\title{
DYKAT of Vinyl Aziridines: Total Synthesis of (+)-Pseudodistomin D
}

\author{
Barry M. Trost and Daniel R. Fandrick \\ Department of Chemistry, Stanford University, Stanford, California 94305-5080 \\ bmtrost@stanford.edu
}

\author{
Supporting Information \\ Part A: Experimental Procedures
}

\section{General}

Flash chromatography was preformed on silica gel (EM Science, Kieselgel 60, 230400mesh, ASTM) or neutral alumina (Fluka, Aluminum Oxide, type 507, Brockmann grade III, $6 \%$ hydrate) using compressed air. TLC was preformed using glass-backed plates coated with $0.2 \mathrm{~mm}$ silica (Merck, DC-Platten, Kieselgel; $60 \mathrm{~F}_{254}$ ) or plastic backed plates coated with $0.2 \mathrm{~mm}$ neutral alumina (EM, $60 \mathrm{~F}_{254}$, Type E). Chiral HPLC analysis was preformed on Daicel Chiralpack columns, eluting with a heptane and isopropanol mixture, using a Thermo Separation Products Spectra SERIES P100 or P200 instruments. NMR spectra were carried out on a Varian Gemini 300 (300 MHz), Mercury $400(400 \mathrm{MHz})$, or Unity $500(500 \mathrm{MHz})$ instruments and are calibrated to TMS or residual solvent peaks: proton $\left(\mathrm{CDCl}_{3} 7.26 \mathrm{ppm}, \mathrm{MeOH} 4.87 \mathrm{ppm}\right)$ and carbon $\left(\mathrm{CDCl}_{3} 77 \mathrm{ppm}, \mathrm{MeOH} 49.15 \mathrm{ppm}\right)$. Optical rotation was measured on a Jasco DIP-1000 digital polarimeter in $5 \mathrm{~cm}$ cells at room temperature. $\left({ }^{3}-\mathrm{C}_{3} \mathrm{H}_{5} \mathrm{PdCl}\right)_{2},{ }^{1}$ Trost ligand $(\mathrm{S}, \mathrm{S}) \mathrm{L}_{\mathrm{N}},{ }^{2}$ alkyne $\mathbf{8}^{3}$ and vinyl iodide $\mathbf{9}^{4}$ were prepared according to literature procedures. All compounds are $>95 \%$ pure by proton NMR unless otherwise noted. All reagents were used as purchased unless otherwise noted.

\section{Experimental Procedures}

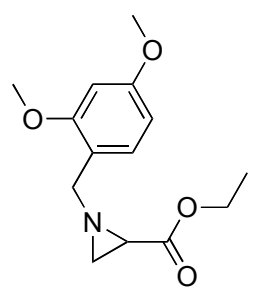

\section{1-(2,4-Dimethoxybenzyl)-aziridine-2-carboxylic acid ethyl ester}

2,4 Dimethoxybenzylamine $(5.0 \mathrm{~mL}, 5.7 \mathrm{~g}, 33 \mathrm{mmol})$ followed by $\mathrm{Et}_{3} \mathrm{~N}(67$ mmol, $6.7 \mathrm{~g}, 9.3 \mathrm{~mL}$ ) were added to a solution of ethyl 2,3-dibromopropanoate 5 (4.8 $\mathrm{mL}, 8.7 \mathrm{~g}, 33 \mathrm{mmol})$ in anhydrous ethanol $(300 \mathrm{~mL})$ at $0^{\circ} \mathrm{C}$ under nitrogen. The reaction was allowed to warm to room temperature and stirred at $50^{\circ} \mathrm{C}$ in a sealed flask of nitrogen for $20 \mathrm{~h}$. The resulting yellow solution was concentrated in vacuo to an oily solid. The oily solid was suspended in ether $(300 \mathrm{~mL})$ and vigorously stirred for $\_$h. The solids were removed by filtration and rinsed thoroughly with ether. The filtrate was

\footnotetext{
${ }^{1}$ Inorganic Syntheses 1991, 28, 342-343.

${ }^{2}$ Trost, B.M.; Bunt, R.C.; Lemoine, R.C.; Calkins, T.L. J. Am. Chem. Soc. 2000, 122, 5968-5976.

3 a) Ma, D.; Lu, X. Tetrahedron 1990, 46, 6319-6330. b) Huang, H.; Forsyth, C.J. J. Org. Chem. 1997, 62, 8595-8599.

${ }^{4}$ Chen, M.-J.; Narkunan, K.; Liu, R.-S. J. Org. Chem. 1999, 64, 8311-8318.
} 
concentrated in vacuo to afford the intended aziridine as a yellow oil $(8.5 \mathrm{~g}, 96 \%)$. This material was used in the next step without further purification. ${ }^{1} \mathbf{H}$ NMR $\left(\mathrm{CDCl}_{3} 300\right.$ MHz) $7.32(\mathrm{~d}, J=8.1 \mathrm{~Hz}, 1 \mathrm{H}), 6.47(\mathrm{dd}, J=8.4,2.1 \mathrm{~Hz}, 1 \mathrm{H}), 6.43(\mathrm{~d}, J=2.4 \mathrm{~Hz}, 1 \mathrm{H})$, 4.12-4.25 (m, 2H), $3.80(\mathrm{~s}, 3 \mathrm{H}), 3.79(\mathrm{~s}, 3 \mathrm{H}), 3.55(\mathrm{~d}, J=14 \mathrm{~Hz}, 1 \mathrm{H}), 3.47$ (d, $J=14 \mathrm{~Hz}$, $1 \mathrm{H}), 2.19-2.20(\mathrm{~m}, 2 \mathrm{H}), 1.76(\mathrm{dd}, J=4.4,3.2 \mathrm{~Hz}, 1 \mathrm{H}), 1.27(\mathrm{t}, J=7.2 \mathrm{~Hz}, 3 \mathrm{H}) .{ }^{13} \mathbf{C}$ NMR $\left(\mathrm{CDCl}_{3} 100 \mathrm{MHz}\right)_{-} 171.03,160.06,157.98,130.03,118.54,103.82,98.11,60.89,57.44$, 55.24, 55.11, 37.35, 34.21, 14.10. IR (KBr -Neat) 2979, 2837, 1742, 1614, 1589, 1509, $1465,1415,1385,1289,1209,1187,1157,1121,1083,1038,834 \mathrm{~cm}^{-1}$. EI-HRMS calculated for $\mathrm{C}_{14} \mathrm{H}_{19} \mathrm{NO}_{4} 265.1314$ found 265.1311 .

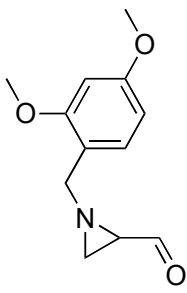

\section{1-(2,4-Dimethoxybenzyl)-aziridine-2-carbaldehyde}

DIBAL-H (1M in Hexanes, $63 \mathrm{~mL}, 63 \mathrm{mmol})$ was slowly added over 20 minutes to an anhydrous solution of 1-(2,4-dimethoxybenzyl)-aziridine-2-carboxylic acid ethyl ester $(7.95 \mathrm{~g}, 30.0 \mathrm{mmol})$ in methylene chloride $(125 \mathrm{~mL})$ at $-78^{\circ} \mathrm{C}$ under nitrogen. The solution was stirred for $1.5 \mathrm{~h}$ at $-78^{\circ} \mathrm{C}$. The reaction was carefully quenched with a saturated aqueous solution of $\mathrm{NaF}(38 \mathrm{~mL})$ at $-78^{\circ} \mathrm{C}$. The resulting mixture was vigorously stirred for $50 \mathrm{~min}$ while allowing the reaction to warm to room temperature. The mixture was poured into methylene chloride $(750 \mathrm{~mL})$, dried with $\mathrm{Na}_{2} \mathrm{SO}_{4}$, filtered, and concentrated in vacuo to provide the intended aldehyde as a cloudy orange oil. The aldehyde was isolated in $>95 \%$ purity by ${ }^{1} \mathrm{H}$ NMR and used in the next reaction without further purification $(6.4 \mathrm{~g}, 97 \%){ }^{1} \mathbf{H}$ NMR $\left(\mathrm{CDCl}_{3} 300 \mathrm{MHz}\right) \_8.90(\mathrm{~d}, J=6.6 \mathrm{~Hz}, 1 \mathrm{H})$, $7.23(\mathrm{~d}, J=8.1 \mathrm{~Hz}, 1 \mathrm{H}), 6.45-6.49(\mathrm{~m}, 2 \mathrm{H}), 3.80(\mathrm{~s}, 3 \mathrm{H}), 3.80(\mathrm{~s}, 3 \mathrm{H}), 3.52(\mathrm{~s}, 2 \mathrm{H}), 2.18$ $2.26(\mathrm{~m}, 2 \mathrm{H}), 1.96(\mathrm{~d}, J=6.6 \mathrm{~Hz}, 1 \mathrm{H}) .{ }^{13} \mathbf{C}$ NMR $\left(\mathrm{CDCl}_{3} 75 \mathrm{MHz}\right) \ldots 200.35,160.38$, $158.16,130.35,118.29,104.02,98.39,57.15,55.29,55.25,44.59,32.52$. IR ( $\mathrm{KBr}-\mathrm{Neat})$ 2939, 2837, 1719, 1614, 1589, 1509, 1464, 1290, 1266, 1209, 1158, 1122, 1035, $834 \mathrm{~cm}^{-}$

${ }^{1}$. EI-HRMS calculated for $\mathrm{C}_{12} \mathrm{H}_{15} \mathrm{NO}_{3} 221.1052$ found 221.1049.

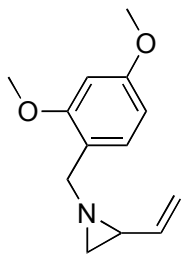

\section{1-(2,4-Dimethoxybenzyl)-2-vinyl-aziridine (4)}

KHMDS (0.47 M in THF, $86 \mathrm{~mL}, 40 \mathrm{mmol}$ ) was slowly added over 20 minutes to an anhydrous suspension of methyltriphenylphosphonium bromide (16 g, $45 \mathrm{mmol})$ in THF $(150 \mathrm{~mL})$ at $-15^{\circ} \mathrm{C}$ under nitrogen. The resulting heterogeneous yellow mixture was stirred at $-15^{\circ} \mathrm{C}$ for $15 \mathrm{~min}$ and at $0^{\circ} \mathrm{C}$ for $1 \mathrm{~h}$. An anhydrous solution of 1-(2,4-dimethoxybenzyl)-aziridine-2-carbaldehyde ( $6.4 \mathrm{~g}, 29 \mathrm{mmol})$ in THF $(70 \mathrm{~mL})$ was added to the above mixture over $10 \mathrm{~min}$ at $-15^{\circ} \mathrm{C}$. The yellow mixture was stirred at $-15^{\circ} \mathrm{C}$ for $15 \mathrm{~min}$, warmed to ambient temperature, and stirred at for $45 \mathrm{~min}$. The yellow mixture was 
poured into a mixture of brine $(100 \mathrm{~mL})$ and ether $(100 \mathrm{~mL})$. The layers were separated, and the aqueous portion was extracted with $3 \times 100 \mathrm{~mL}$ of ether. The combined organic extracts were dried with $\mathrm{Na}_{2} \mathrm{SO}_{4}$, filtered, and concentrated in vacuo to yield a cloudy, thick, and orange oil. The intended vinyl aziridine 4 was isolated in $>95 \%$ purity as a clear and slightly yellow oil $(4.8 \mathrm{~g}, 76 \%)$ after purification by silica gel chromatography $\left(50 \% \mathrm{Et}_{2} \mathrm{O}\right.$ in petroleum ether). ${ }^{1} \mathbf{H}$ NMR $\left(\mathrm{CDCl}_{3} 400 \mathrm{MHz}\right) \quad 7.33(\mathrm{~d}, J=8.3 \mathrm{~Hz}, 1 \mathrm{H})$, 6.47 (dd, $J=8.3,2.4 \mathrm{~Hz}, 1 \mathrm{H}), 6.44$ (d, $J=2.4 \mathrm{~Hz}, 1 \mathrm{H}), 5.59$ (ddd, $J=17.2,10.3,8.0 \mathrm{~Hz}$, $1 \mathrm{H}), 5.30$ (ddd, $J=17.2,1.6,0.6 \mathrm{~Hz}, 1 \mathrm{H}), 5.11$ (ddd, $J=10.3,1.6,0.5 \mathrm{~Hz}, 1 \mathrm{H}), 3.79$ (s, $3 \mathrm{H}), 3.79$ (s, 3H), 3.51 (d, $J=14 \mathrm{~Hz}, 1 \mathrm{H}), 3.37$ (d, $J=14 \mathrm{~Hz}, 1 \mathrm{H}), 2.01$ (ddd, $J=7.9,6.5$, $3.5 \mathrm{~Hz}, 1 \mathrm{H}), 1.83(\mathrm{~d}, J=3.4 \mathrm{~Hz}, 1 \mathrm{H}), 1.65(\mathrm{~d}, J=6.5 \mathrm{~Hz}, 1 \mathrm{H}) .{ }^{13} \mathbf{C} \mathbf{N M R}\left(\mathrm{CDCl}_{3} 100\right.$ MHz)_159.81, 157.83, 138.71, 129.50, 119.96, 115.97, 103.76, 98.15, 58.25, 55.28, 55.19, 41.36, 35.28. IR (KBr -Neat) 2958, 2836, 1614, 1590, 1508, 1464, 1438, 1418, $1288,1258,1209,1156,1121,1038,918,833,733 \mathrm{~cm}^{-1}$. EI-HRMS calculated for $\mathrm{C}_{13} \mathrm{H}_{17} \mathrm{NO}_{2} 219.1259$ found 219.1260 .

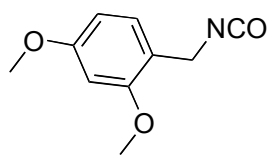

\section{2,4 Dimethoxybenzyl isocyanate}

2.4-Dimethoxybenzyl isocyanate was prepared by a modification of the procedure published by Hirota et. al..$^{5}$ An anhydrous solution of 2,4-dimethoxybenzylamine (1.97 $\mathrm{mL}, 2.20 \mathrm{~g}, 13 \mathrm{mmol})$ and $\mathrm{Et}_{3} \mathrm{~N}(3.66 \mathrm{~mL}, 2.66 \mathrm{~g}, 26.3 \mathrm{mmol})$ in ether $(100 \mathrm{~mL})$ was carefully added through an addition funnel to an anhydrous solution of triphosgene (1.30 $\mathrm{g}, 4.4 \mathrm{mmol})$ in ether $(50 \mathrm{~mL})$ at $-78^{\circ} \mathrm{C}$ under nitrogen. After stirring for $10 \mathrm{~min}$, the heterogeneous solution was allowed to warm to ambient temperature over $1 \mathrm{~h}$. The mixture was then diluted with ether $(100 \mathrm{~mL})$, and the solids were removed by filtration. The filtrate was concentrated in vacuo to a yellow oil. The crude oil was distilled to afford the intended isocyanate as a cloudy colorless oil $(1.86 \mathrm{~g}, 74 \%) .{ }^{\mathbf{1}} \mathbf{H ~ N M R}\left(\mathrm{CDCl}_{3}\right.$ $400 \mathrm{MHz})_{-} 7.15(\mathrm{~d}, J=7.6 \mathrm{~Hz}, 1 \mathrm{H}), 6.40-6.50(\mathrm{~m}, 2 \mathrm{H}), 4.33(\mathrm{~s}, 2 \mathrm{H}), 3.84(\mathrm{~s}, 3 \mathrm{H}), 3.81(\mathrm{~s}$, $3 \mathrm{H}) .{ }^{13} \mathbf{C} \mathbf{N M R}\left(\mathrm{CDCl}_{3} 100 \mathrm{MHz}\right) \ldots 160.96,158.09,129.46,124.56,117.93,103.89$, 98.58, 55.38, 55.31, 42.25. IR (KBr-Neat) 3005, 2941, 2838, 2251 (s), 1614, 1590, $1509,1465,1439,1419,1294,1267,1210,1158,1131,1036,921,828,781 \mathrm{~cm}^{-1}$. EIHRMS calculated for $\mathrm{C}_{10} \mathrm{H}_{11} \mathrm{NO}_{3} 193.0793$ found 193.0732.

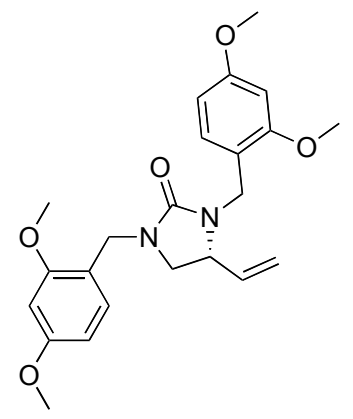

(R)-(+)-1,3-Bis-(2,4-dimethoxybenzyl)-4-vinyl-imidazolidin-2-one (3)

\footnotetext{
${ }^{5}$ Hirota, K.; Kazaoka, K.; Niimoto, I.; Sajiki, H. Org. Biomol. Chem. 2003, 1, 1354-1365.
} 
Degassed and anhydrous methylene chloride $(90 \mathrm{~mL})$ was added to a flame dried $250 \mathrm{~mL}$ round bottom flask charged with $\left({ }^{3}-\mathrm{C}_{3} \mathrm{H}_{5} \mathrm{PdCl}\right)_{2}(85.3 \mathrm{mg}, 235$ mol $)$ and $(\mathrm{S}, \mathrm{S})$ $\mathrm{L}_{\mathrm{N}}(560 \mathrm{mg}, 0.71 \mathrm{mmol})$ under nitrogen. The solution was stirred for $15 \mathrm{~min}$ to afford a deep red homogeneous solution to which was added acetic acid (67_L, $71 \mathrm{mg}, 1.18$ mmol). After stirring for another $10 \mathrm{~min}$, aziridine 4 (2.58 g, $11.8 \mathrm{mmol})$ and 2,4 dimethoxybenzyl isocyanate $(2.38 \mathrm{~g}, 12.3 \mathrm{mmol})$ were added in sequence to the above catalyst solution in a room temperature water bath, to control the exothermic additions. The resulting yellow homogeneous solution was stirred under nitrogen for $18 \mathrm{~h}$ to afford a deep red orange solution. At which point, all aziridine was absent by TLC. The reaction was concentrated in vacuo to provide a red oil. The oil was purified by silica gel chromatography ( $45 \%$ ethyl acetate in petroleum ether) to afford the intended imidazolidinone 3 as a tan viscous oil $(4.28 \mathrm{~g}, 88 \%)$. [ $]_{\mathbf{D}}{ }^{22.2}=+15.6^{\circ}\left(\mathrm{c}=1.19\right.$ in $\mathrm{CHCl}_{3}$ at $94 \%$ ee) Chiralpak OJ HPLC (10\% isopropanol in Heptane, $1 \mathrm{~mL} / \mathrm{min}, 230 \mathrm{~nm}$ ) shows 94\% ee favoring 23.718 over 20.423 min compared to a racemic sample ${ }^{6}$ that shows enantiomers at 19.248 and $22.875 \mathrm{~min} .{ }^{1} \mathbf{H}$ NMR $\left(\mathrm{CDCl}_{3} 300 \mathrm{MHz}\right){ }_{-} 7.20(\mathrm{~d}, J=7.8 \mathrm{~Hz}$, $1 \mathrm{H}), 7.19(\mathrm{~d}, J=8.1 \mathrm{~Hz}, 1 \mathrm{H}), 6.40-6.46(\mathrm{~m}, 4 \mathrm{H}), 5.65$ (ddd, $J=17.1,10.1,8.5 \mathrm{~Hz}, 1 \mathrm{H})$, $5.14(\mathrm{~d}, J=8.4 \mathrm{~Hz}, 1 \mathrm{H}), 5.10(\mathrm{~d}, J=16.5 \mathrm{~Hz}, 1 \mathrm{H}), 4.59$ (d, $J=15.3 \mathrm{~Hz}, 1 \mathrm{H}), 4.41$ (d, $J=14.7$ $\mathrm{Hz}, 1 \mathrm{H}), 4.31$ (d, $J=15.0 \mathrm{~Hz}, 1 \mathrm{H}), 4.08$ (d, $J=15.0 \mathrm{~Hz}, 1 \mathrm{H}), 3.70-3.82$ (m, 13H), 3.31 (t, $J=8.7 \mathrm{~Hz}, 1 \mathrm{H}), 2.86(\mathrm{dd}, J=9.0,7.5 \mathrm{~Hz}, 1 \mathrm{H}) .{ }^{13} \mathbf{C ~ N M R}\left(\mathrm{CDCl}_{3} 75 \mathrm{MHz}\right){ }_{-} 160.78$, $160.14,160.00,158.55,158.39,136.91,130.73,130.54,118.30,118.29,117.94,104.11$, 103.92, 98.20, 98.08, 56.20, 55.25, 55.05, 48.63, 41.91, 39.57. IR (KBr -Neat) 2937, 2837, 1694 (s), 1612, 1589, 1508, 1453, 1421, 1294, 1240, 1210, 1157, 1121, 1037, 920, $834,732 \mathrm{~cm}^{-1}$. EI-HRMS calculated for $\mathrm{C}_{23} \mathrm{H}_{28} \mathrm{~N}_{2} \mathrm{O}_{5} 412.1998$ found 412.2019.

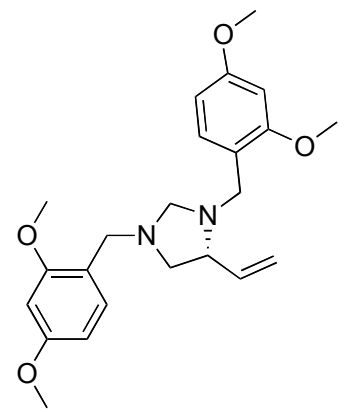

\section{(R)-(-)-1,3-Bis-(2,4-dimethoxybenzyl)-4-vinyl-imidazolidine}

Solid $\mathrm{LiAlH}_{4}(737 \mathrm{mg}, 19.4 \mathrm{mmol})$ was added in small portions to an anhydrous solution of imidazolidinone $3(2.0 \mathrm{~g}, 4.85 \mathrm{mmol})$ in ether $(200 \mathrm{~mL})$. The gray suspension was stirred at reflux for $13 \mathrm{~h}$ at which point starting imidazolidinone $\mathbf{3}$ was still present by TLC. An additional $250 \mathrm{mg}$ of $\mathrm{LiAlH}_{4}$ were added to the above reaction, and the suspension was stirred at reflux for another $6 \mathrm{~h}$ at which point all starting imidazolidinone was absent by TLC. The reaction was carefully quenched at $0^{\circ} \mathrm{C}$ by the consecutive addition of water $(1 \mathrm{~mL}), 4 \mathrm{M} \mathrm{NaOH}(1 \mathrm{~mL})$, and water $(2.2 \mathrm{~mL})$. After stirring the resulting mixture for $\mathrm{h}_{\mathrm{h}}$ at $0^{\circ} \mathrm{C}$ and $\mathrm{h}_{\mathrm{h}} \mathrm{h}$ at ambient temperature, the mixture was diluted with methylene chloride $(150 \mathrm{~mL})$, dried with $\mathrm{Na}_{2} \mathrm{SO}_{4}$, filtered, and concentrated to provide the intended imidazolidine as a yellow oil (1.92 g) in $90-95 \%$ purity by ${ }^{1} \mathrm{H} \mathrm{NMR}$.

\footnotetext{
${ }^{6}$ Racemic imidazolidinone 3 was prepared by the same procedure as that for chiral $\mathbf{3}$ but $12 \%$ triphenylphosphine was employed as the ligand.
} 
This material was used in the next step without further purification. [_l $\mathbf{D}^{24.1}=-59.0^{\circ}$ (c=1.32 in $\mathrm{CHCl}_{3}$ at $94 \%$ ee) ${ }^{1} \mathbf{H} \mathbf{~ N M R}\left(\mathrm{CDCl}_{3} 400 \mathrm{MHz}\right) \ldots 7.28(\mathrm{~d}, \bar{J}=8.4 \mathrm{~Hz}, 1 \mathrm{H}), 7.22$ $(\mathrm{d}, J=8.5 \mathrm{~Hz}, 1 \mathrm{H}), 6.40-6.46(\mathrm{~m}, 4 \mathrm{H}), 5.81$ (ddd, $J=17.2,10.1,7.9 \mathrm{~Hz}, 1 \mathrm{H}), 5.22$ (dd, $J=17.1,1.9,0.8 \mathrm{~Hz}, 1 \mathrm{H}), 5.09$ (ddd, $J=10.1,1.9,0.6 \mathrm{~Hz}, 1 \mathrm{H}), 3.75-3.84$ (m, $13 \mathrm{H}), 3.73$ $(\mathrm{d}, J=5.9 \mathrm{~Hz}, 1 \mathrm{H}), 3.66$ (s, 2H), 3.47 (d, $J=13.9 \mathrm{~Hz}, 1 \mathrm{H}), 3.34$ (d, $J=5.9 \mathrm{~Hz}, 1 \mathrm{H}), 3.27$ (quartet, $J=7.9 \mathrm{~Hz}, 1 \mathrm{H}), 3.07(\mathrm{dd}, J=9.6,7.3 \mathrm{~Hz}, 1 \mathrm{H}), 2.64(\mathrm{dd}, J=9.6,8.2 \mathrm{~Hz}, 1 \mathrm{H}) .{ }^{13} \mathbf{C}$ NMR $\left(\mathrm{CDCl}_{3}, 100 \mathrm{MHz}\right)_{-}$159.73, 159.64, 158.32, 158.30, 139.40, 130.61, 130.51, $120.09,119.85,116.41,103.77,103.75,98.27,98.20,76.30,66.80,58.79,55.30,55.27$, 55.24, 53.06, 49.88. IR (KBr-Neat) 2937, 2834, 1614, 1589, 1507, 1485, 1438, 1419, $1290,1265,1208,1156,1132,1039,920,833,733 \mathrm{~cm}^{-1}$. EI-HRMS calculated for $\mathrm{C}_{23} \mathrm{H}_{30} \mathrm{~N}_{2} \mathrm{O}_{4} 398.2206$ found 398.2184.

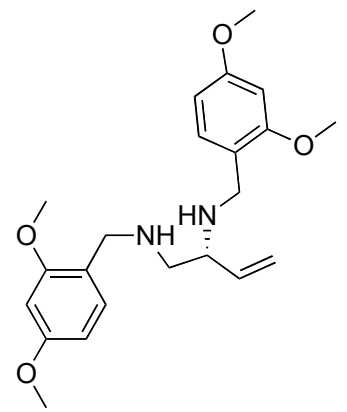

(R)-(-)- $N^{\mathbf{1}}, N^{\mathbf{2}}$-Bis-(2,4-dimethoxybenzyl)-but-3-ene-1,2-diamine

$\mathrm{H}_{2} \mathrm{NOH}-\mathrm{HCl}$ (1.69 g, $24.3 \mathrm{mmol}$ ) was added as a solid to a suspension of $(R)-(-)-$ 1,3-bis-(2,4-dimethoxybenzyl)-4-vinylimidazolidine (all material from above) in aqueous $0.01 \% \mathrm{HCl}(125 \mathrm{~mL})$. The suspension was stirred at $60^{\circ} \mathrm{C}$ for $1 \mathrm{~h}$ to afford a homogeneous colorless solution with no starting material present by TLC. After cooling the reaction to room temperature, the solution was diluted with $0.5 \mathrm{M} \mathrm{HCl}$ aq. $(100 \mathrm{~mL})$

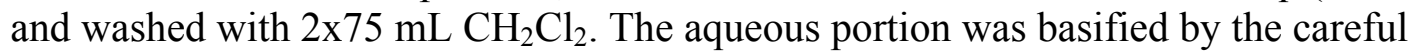
addition of solid $\mathrm{Na}_{2} \mathrm{CO}_{3}$ and $1 \mathrm{M} \mathrm{NaOH}(50 \mathrm{~mL})$. The resulting aqueous suspension was extracted with $4 \times 75 \mathrm{~mL}$ of $\mathrm{CH}_{2} \mathrm{Cl}_{2}$. The final basic organic extracts were dried with $\mathrm{Na}_{2} \mathrm{SO}_{4}$, filtered, and concentrated to the intended diamine (1.72 $\mathrm{g}, 92 \%$ for two steps) in approximately $95 \%$ purity by ${ }^{1} \mathrm{H}$ NMR analysis. This material was used in the next step without further purification. [_] ${ }^{25.7}=-19.0^{\circ}\left(\mathrm{c}=2.20\right.$ in $\mathrm{CHCl}_{3}$ at $94 \%$ ee ${ }^{\mathbf{1}} \mathbf{H} \mathbf{~ N M R}$ $\left(\mathrm{CDCl}_{3} 300 \mathrm{MHz}\right)_{-} 7.11(\mathrm{~d}, J=8.1 \mathrm{~Hz}, 1 \mathrm{H}), 7.08(\mathrm{~d}, J=7.8 \mathrm{~Hz}, 1 \mathrm{H}), 6.38-6.42(\mathrm{~m}, 4 \mathrm{H})$, 5.66 (ddd, $J=17.7,10.2,7.8 \mathrm{~Hz}, 1 \mathrm{H}), 5.19$ (d, $J=16.2 \mathrm{~Hz}, 1 \mathrm{H}), 5.15(\mathrm{~d}, J=10.2 \mathrm{~Hz}, 1 \mathrm{H})$, $3.50-3.86(\mathrm{~m}, 16 \mathrm{H}), 3.17$ (quartet, $J=7.5 \mathrm{~Hz}, 1 \mathrm{H}), 2.52-2.66(\mathrm{~m}, 2 \mathrm{H}), 1.50-2.10(\mathrm{~s}, 2 \mathrm{H})$. ${ }^{13} \mathbf{C ~ N M R}\left(\mathrm{CDCl}_{3}, 75 \mathrm{MHz}\right) \ldots 159.84,158.62,158.48,140.23,130.35,130.14,121.17$, $121.05,116.62,103.49,98.42,98.37,60.09,55.29,55.14,53.20,48.28,46.18$. IR ( $\mathrm{KBr}$ -Neat) 3314, 3000, 2936, 2835, 1613, 1588, 1507, 1464, 1418, 1289, 1260, 1208, 1156 , $1133,1038,921,833,755 \mathrm{~cm}^{-1}$. EI-HRMS calculated for $\mathrm{C}_{22} \mathrm{H}_{30} \mathrm{~N}_{2} \mathrm{O}_{4} 386.2206$ found 386.2219 . 


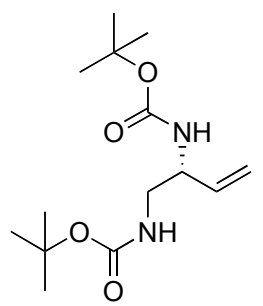

(R)-(+)-1,2 Bis-(tert-butoxycarbonylamino)-3-butene (6)

A mixture of $(R)-(-)-N^{1}, N^{2}$-Bis-(2,4-dimethoxybenzyl)-but-3-ene-1,2-diamine $(1.77 \mathrm{~g}, 4.59 \mathrm{mmol})$ and $\mathrm{TsOH}-\mathrm{H}_{2} \mathrm{O}(6.98 \mathrm{~g}, 36.7 \mathrm{mmol})$ in toluene $(13 \mathrm{~mL})$ was stirred at $110^{\circ} \mathrm{C}$ in a sealed tube under argon for $7 \mathrm{~h}$ to afford a biphasic deep purple mixture. The mixture was cooled to room temperature and diluted with methanol $(63 \mathrm{~mL})$ and $\mathrm{Et}_{3} \mathrm{~N}(7 \mathrm{~mL})$. Di-tert-butyl-dicarbonate $(5.0 \mathrm{~g}, 23 \mathrm{mmol})$ was added to the resulting tan mixture. The mixture was stirred at ambient temperature for $45 \mathrm{~min}$ and at $60^{\circ} \mathrm{C}$ for $2.5 \mathrm{~h}$. The mixture was cooled to room temperature, and approximately $90 \%$ of the solvent was removed in vacuo. The brown concentrate was suspended in ethyl acetate $(170 \mathrm{~mL})$ and washed with $3 \times 50 \mathrm{~mL} 0.5 \mathrm{M} \mathrm{pH}=5$ phosphate buffer, $2 \times 50 \mathrm{~mL}$ aqueous and saturated $\mathrm{NaHCO}_{3}, 1 \times 50 \mathrm{~mL}$ brine, dried with $\mathrm{Na}_{2} \mathrm{SO}_{4}$, filtered, and concentrated to a tan solid. Purification by silica gel chromatography (30\% ethyl acetate in petroleum ether) and recrystallization from hot heptane afforded diamine 6 as thin white needles $(730 \mathrm{mg}$, $56 \%) . \mathbf{M p}=116-118^{\circ} \mathrm{C}$. [_] ${ }^{24.9}=+26.2^{\circ}\left(\mathrm{c}=1.09\right.$ in $\left.\mathrm{CDCl}_{3}\right) .{ }^{1} \mathbf{H}$ NMR $\left(\mathrm{DMSO}-\mathrm{d}^{6}, 80^{\circ} \mathrm{C}\right.$, $300 \mathrm{MHz}{ }_{-} 6.40(\mathrm{bs}, 2 \mathrm{H}), 5.74(\mathrm{ddd}, J=17.1,10.5,6.0 \mathrm{~Hz}, 1 \mathrm{H}), 5.11(\mathrm{dt}, J=17.1,1.8 \mathrm{~Hz}$, $1 \mathrm{H}), 5.05(\overline{\mathrm{dt}}, J=10.5,1.2 \mathrm{~Hz}, 1 \mathrm{H}), 4.05(\mathrm{q}, J=6.6 \mathrm{~Hz}, 1 \mathrm{H}), 3.00-3.06(\mathrm{~m}, 2 \mathrm{H}), 1.40(\mathrm{~s}$, 9H), 1.39 (s, 9H). ${ }^{13} \mathrm{C}$ NMR (DMSO-d $\left.{ }^{6}, 80^{\circ} \mathrm{C}, 75 \mathrm{MHz}\right) \ldots 155.29,154.63,137.09$, 114.56, 77.39, 52.66, 43.60, 27.83. IR (KBr-neat) 3362, 2986, 2937, 1686 (s), 1531 (s), $1447,1390,1367,1310,1274,1251,1225,1170,1041,1016,994,920,882 \mathrm{~cm}^{-1}$. EA calculated for $\mathrm{C}_{14} \mathrm{H}_{26} \mathrm{~N}_{2} \mathrm{O}_{4} \mathrm{C}: 58.72, \mathrm{H}: 9.15$ found $\mathrm{C}: 58.80, \mathrm{H}: 9.30$.

\section{Diastereoselective Epoxidation of Olefin 6:}

A mixture of olefin $6(1.63 \mathrm{~g}, 5.70 \mathrm{mmol})$, purified $\mathrm{mCPBA}^{7}(4.92 \mathrm{~g}, 28.5 \mathrm{mmol})$ and $\mathrm{NaHCO}_{3}(2.40 \mathrm{~g}, 28.5 \mathrm{mmol})$ in anhydrous $\mathrm{CH}_{2} \mathrm{Cl}_{2}(100 \mathrm{~mL})$ was stirred at reflux for $5 \mathrm{~h}$ at which point all starting material was consumed by TLC. The reaction was cooled to room temperature and diluted with a solution of $1 \mathrm{M} \mathrm{Na}_{2} \mathrm{SO}_{3}(40 \mathrm{~mL})$ and saturated $\mathrm{NaHCO}_{3}(40 \mathrm{~mL})$. After stirring the bubbling mixture room temperature for $1 / 2 \mathrm{~h}$, it was extracted with $1 \times 100 \mathrm{~mL} \mathrm{CH} \mathrm{Cl}_{2}$. The organic extract was washed with $1 \times 100 \mathrm{~mL}(50$ $\mathrm{mL} 1 \mathrm{M} \mathrm{Na}_{2} \mathrm{SO}_{3}, 50 \mathrm{~mL}$ saturated $\left.\mathrm{NaHCO}_{3}\right), 1 \times 100 \mathrm{~mL}\left(75 \mathrm{~mL}\right.$ saturated $\mathrm{NaHCO}_{3}, 25$ $\mathrm{mL}$ brine), $1 \times 100 \mathrm{~mL}$ brine, dried with $\mathrm{Na}_{2} \mathrm{SO}_{4}$, filtered, and concentrated to a white solid. Crude ${ }^{1} \mathrm{H}$ NMR analysis $\left(\mathrm{CDCl}_{3}, 55^{\circ} \mathrm{C}\right)$ showed a 4:1 diastereomeric ratio in favor diastereomer 7. Purification by silica gel chromatography (30\% ethyl acetate in petroleum ether) provided the diastereomeric epoxides in a combined $84 \%$ yield.

\footnotetext{
${ }^{7}$ Perrin, D.D.; Armarego, L.F. Purification of Laboratory Chemicals, $3^{\text {rd }}$ ed.; Pergamon Press: Elmsford, NY, 1988, pg 123.
} 


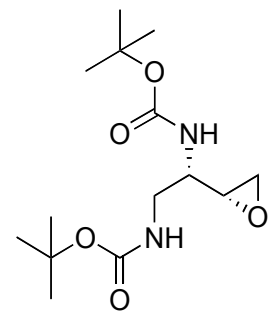

(2S,3R)-(+)-1,2-Bis-(tert-Butoxycarbonylamino)-3,4-epoxybut-3 ene (threo epoxide) (7) White powder (1.15 g, 67\%). Mp = $135-140^{\circ} \mathrm{C}$. [_] ${ }_{\mathbf{D}}^{23.2}=+16.6^{\circ}\left(\mathrm{c}=0.82\right.$ in $\left.\mathrm{CHCl}_{3}\right) .{ }^{1} \mathbf{H}$ NMR $\left(\mathrm{CDCl}_{3}\right.$, $\left.55^{\circ} \mathrm{C}, 500 \mathrm{MHz}\right)_{-} 4.81(\mathrm{~s}, 1 \mathrm{H}), 4.62(\mathrm{~s}, 1 \mathrm{H}), 3.96(\mathrm{~m}, 1 \mathrm{H}), 3.30-$ $3.41(\mathrm{~m}, 2 \mathrm{H}), 3.06(\mathrm{dt}, J=4.0,2.6 \mathrm{~Hz}, 1 \mathrm{H}), 2.72(\mathrm{dd}, J=4.8,4.0 \mathrm{~Hz}$, $1 \mathrm{H}), 2.59$ (dd, $J=4.8,2.7 \mathrm{~Hz}, 1 \mathrm{H}), 1.45(\mathrm{~s}, 9 \mathrm{H}), 1.43(\mathrm{~s}, 9 \mathrm{H}) .{ }^{13} \mathrm{C}$ NMR $\left(\mathrm{CDCl}_{3}, 50^{\circ} \mathrm{C}, 75 \mathrm{MHz}\right)_{-} 156.20,155.84,79.87,79.65$, 51.80, 50.01, 43.66, 43.42, 28.37, 28.33. IR (KBr-neat) 3356, 2987, 2940, 1685 (s), 1528, 1459, 1392, 1369, 1318, 1276, 1249, 1223, 1161, 1018, 872 $\mathrm{cm}^{-1}$. EI-HRMS $\mathrm{C}_{14} \mathrm{H}_{26} \mathrm{~N}_{2} \mathrm{O}_{5}(\mathrm{M})^{+}$, calculated for $\left(\mathrm{M}-\mathrm{C}_{4} \mathrm{H}_{9}\right)^{+} 245.1137$ found 245.1165, calculated for $\left(\mathrm{M}-\mathrm{C}_{4} \mathrm{H}_{9} \mathrm{O}\right)^{+} 229.1188$ found 229.1188 . ESI-MS calculated for $\mathrm{C}_{14} \mathrm{H}_{26} \mathrm{~N}_{2} \mathrm{O}_{5} 302.2$ found $(\mathrm{M}+\mathrm{Na})^{+}$325.2. EA calculated for $\mathrm{C}_{14} \mathrm{H}_{26} \mathrm{~N}_{2} \mathrm{O}_{5}$ : C: 55.61 and $\mathrm{H}$ : 8.67 found $\mathrm{C}: 55.49$ and $\mathrm{H}: 8.45$.

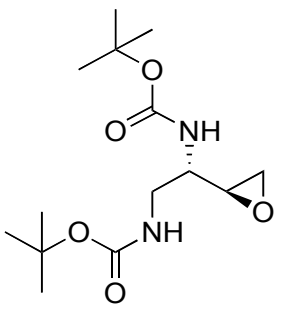

(2S,3S)-(+)-1,2-Bis-(tert-Butoxycarbonylamino)-3,4-epoxy-but3 ene (erythro epoxide) White solid (295 mg, 17\%). Mp $=99$ $102^{\circ} \mathrm{C}$. [ ] ${ }_{\mathbf{D}}{ }^{22.7}=+10.8^{\circ} \mathrm{C}\left(\mathrm{c}=0.622\right.$ in $\left.\mathrm{CHCl}_{3}\right) .{ }^{1} \mathbf{H}$ NMR (DMSO$\left.\mathrm{d}^{6}, 80^{\circ} \mathrm{C}, 500 \mathrm{MHz}\right)_{-} 6.44(\mathrm{bs}, 1 \mathrm{H}), 6.35(\mathrm{bs}, 1 \mathrm{H}), 3.35-3.41(\mathrm{~m}$, $1 \mathrm{H}), 3.07-3.18(\mathrm{~m}, 2 \overline{\mathrm{H}}), 2.86(\mathrm{dt}, J=6.2,3.3 \mathrm{~Hz}, 1 \mathrm{H}), 2.64(\mathrm{t}, J=4.6$ $\mathrm{Hz}, 1 \mathrm{H}), 2.59$ (dd, $J=5.2,2.6 \mathrm{~Hz}, 1 \mathrm{H}), 1.40(\mathrm{~s}, 9 \mathrm{H}), 1.39$ (s, 9H). ${ }^{13}$ C NMR (DMSO-d $\left.{ }^{6}, 80^{\circ} \mathrm{C}, 125 \mathrm{MHz}\right){ }_{-} 155.35,154.82,77.63$, 77.49, 51.83, 51.25, 43.94, 41.40, 27.81, 27.78. IR (KBr-neat) 3366, 2983, 2937, 1689 (s), 1532, 1459, 1392, 1369, 1345, 1316, $1252,1164,1043,868 \mathrm{~cm}^{-1}$. EI-HRMS $\mathrm{C}_{14} \mathrm{H}_{26} \mathrm{~N}_{2} \mathrm{O}_{5}(\mathrm{M})^{+}$, calculated for $\left(\mathrm{M}-\mathrm{C}_{4} \mathrm{H}_{9} \mathrm{O}\right)^{+}$ 229.1188 found 229.1191. ESI-MS calculated for $\mathrm{C}_{14} \mathrm{H}_{26} \mathrm{~N}_{2} \mathrm{O}_{5} 302.2$ found $(\mathrm{M}+\mathrm{H})^{+}$ 303.1 and $(\mathrm{M}+\mathrm{Na})^{+}$325.2. EA calculated for $\mathrm{C}_{14} \mathrm{H}_{26} \mathrm{~N}_{2} \mathrm{O}_{5}: \mathrm{C}: 55.61$ and $\mathrm{H}: 8.67$ found $\mathrm{C}$ : 55.80 and $\mathrm{H}: 8.53$.

Trideca-6,8-dien-1-ol (10)<smiles>CCCCC=CC=CCCCCCCO</smiles>

This cross coupling was preformed by a modified procedure published by Negishi et. al. ${ }^{8}$ A suspension of $\mathrm{Cp}_{2} \mathrm{ZrHCl}^{9}(24 \mathrm{~g}, 94 \mathrm{mmol})$ and alkyne $8(18.1 \mathrm{~g}, 92.3 \mathrm{mmol})$ in anhydrous THF $(450 \mathrm{~mL})$ was stirred under $\mathrm{N}_{2}$ shielded from light for $1 \mathrm{~h}$ to afford a deep green solution. To a separate suspension of $\mathrm{PdCl}_{2}(\mathrm{PhCN})_{2}(1.06 \mathrm{~g}, 2.77 \mathrm{mmol})$ and 1,1' bis-(diphenylphosphino) ferrocene $(3.07 \mathrm{~g}, 5.54 \mathrm{mmol})$ in anhydrous THF $(50 \mathrm{~mL})$ was added DIBAL-H (1M in hexanes, $5.5 \mathrm{~mL}, 5.5 \mathrm{mmol})$ under $\mathrm{N}_{2}$ to afford a deep brownred solution. After stirring the catalyst solution for 15 minutes, vinyl iodide 9 ( $20.4 \mathrm{~g}, 97$ mmol) was added to the above catalyst solution. The vinyl iodide and catalyst solution was cannulated to the above hydrozirconated solution. The reaction was stirred under $\mathrm{N}_{2}$ shielded from light for $22 \mathrm{~h}$. The reaction solution was diluted with petroleum ether (200 $\mathrm{mL}$ ) and passed through a neutral alumina plug (eluting with a further $600 \mathrm{~mL}$ of $20 \%$ $\mathrm{Et}_{2} \mathrm{O}$ in petroleum ether). The filtrate was concentrated to an oily brown solid. The solid was suspended in $300 \mathrm{~mL}$ of $10 \% \mathrm{Et}_{2} \mathrm{O}$ in petroleum ether, filtered through celite, and

\footnotetext{
${ }^{8}$ Negishi, E.; Takahashi, T.; Baba, S.; Van Horn, D.E.; Okukado, N. J. Am. Chem. Soc. 1987, 109, 2393 2401.

${ }^{9}$ The Schwartz's reagent was purchased from Aldrich and used without further purification.
} 
concentrated to the THP adduct of alcohol $\mathbf{1 0}$ as a red oil. A solution of the above red oil and $\mathrm{TsOH}-\mathrm{H}_{2} \mathrm{O}(880 \mathrm{mg}, 4.6 \mathrm{mmol})$ in methanol $(300 \mathrm{~mL})$ was stirred at room temperature for $1.25 \mathrm{~h}$ at which point only trace THP protected alcohol remained by TLC. The reaction was quenched with $\mathrm{Et}_{3} \mathrm{~N}(1.3 \mathrm{~mL})$ and concentrated to a brown oil. The brown oil was purified by silica gel chromatography $\left(30 \% \mathrm{Et}_{2} \mathrm{O}\right.$ in petroleum ether) to afford the intended alcohol $\mathbf{1 0}$ as a clear and slightly yellow oil $(14.7 \mathrm{~g}, 82 \%) .{ }^{1} \mathbf{H}$ NMR $\left(\mathrm{CDCl}_{3} 500 \mathrm{MHz}\right)$ _5.92-6.02 (m, 2H), 5.48-5.60 (m, 2H), $3.64(\mathrm{dd}, J=10.5,6 \mathrm{~Hz}$, 2H), 2.04 (quintet, $J=7.5 \mathrm{~Hz}, 4 \mathrm{H}), 1.50-1.60(\mathrm{~m}, 3 \mathrm{H}), 1.20-1.42(\mathrm{~m}, 8 \mathrm{H}), 0.89(\mathrm{t}$, $J=7.0 \mathrm{~Hz}, 3 \mathrm{H}) .{ }^{13} \mathbf{C} \mathbf{N M R}\left(\mathrm{CDCl}_{3} 100 \mathrm{MHz}\right) \ldots 132.57,131.92,130.54,130.19,62.92$, 32.60, 32.49, 32.24, 31.53, 29.16, 25.26, 22.21, 13.91. IR (KBr-Neat) 3346, 3014, 2956, 2928, 1461, 1378, 1054, 986, $729 \mathrm{~cm}^{-1}$.EA calculated for $\mathrm{C}_{13} \mathrm{H}_{24} \mathrm{O}: \mathrm{C}: 79.53 ; \mathrm{H}: 12.32$ found $\mathrm{C}: 79.70 ; \mathrm{H}: 12.46$.

\section{Trideca-6,8-dienal}

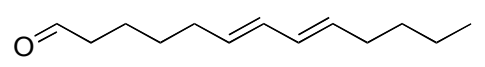

Anhydrous DMSO (9.15 mL, $129 \mathrm{mmol})$ was added dropwise to a anhydrous solution of methylene chloride $(300 \mathrm{~mL})$ and oxalyl chloride $(6.35 \mathrm{~mL}, 73 \mathrm{mmol})$ under nitrogen at $-78^{\circ} \mathrm{C}$. After stirring the solution for $15 \mathrm{~min}$, alcohol 10 (11.0 g, $\left.56.0 \mathrm{mmol}\right)$ dissolved in anhydrous methylene chloride $(200 \mathrm{~mL})$ was cannulated to the above solution at $-78^{\circ} \mathrm{C}$. The solution was stirred at $-78^{\circ} \mathrm{C}$ for $30 \mathrm{~min}$. At which point, $\mathrm{Et}_{3} \mathrm{~N}$ (39 $\mathrm{mL}, 280 \mathrm{mmol}$ ) was added dropwise to the above mixture at $-78^{\circ} \mathrm{C}$. The resulting white mixture was stirred for $2 \mathrm{~h}$ allowing the reaction to warm to room temperature. The resulting yellow-white mixture was diluted with ether $(500 \mathrm{~mL})$, washed with $2 \times 250 \mathrm{~mL}$ $0.5 \mathrm{M} \mathrm{pH}=5$ phosphate buffer, $2 \times 200 \mathrm{~mL}$ saturated $\mathrm{NaHCO}_{3}, 1 \times 150 \mathrm{~mL}$ brine, dried with $\mathrm{Na}_{2} \mathrm{SO}_{4}$, filtered, and concentrated in vacuo to a yellow-brown oil. The product aldehyde was isolated as a yellow oil $(9.75 \mathrm{~g}, 89 \%)$ after silica gel chromatography $(7.5 \%$ $\mathrm{Et}_{2} \mathrm{O}$ in petroleum ether $){ }^{1} \mathbf{H}$ NMR $\left(\mathrm{CDCl}_{3} 400 \mathrm{MHz}\right){ }_{-} 9.76(\mathrm{t}, J=1.6 \mathrm{~Hz}, 1 \mathrm{H}), 5.95-6.02$ (m, 2H), 5.48-5.60 (m, 2H), $2.43(\mathrm{dt}, J=7.6,2.0 \mathrm{~Hz}, 2 \mathrm{H}), 2.02-2.12(\mathrm{~m}, 4 \mathrm{H}), 1.58-1.68$ $(\mathrm{m}, 2 \mathrm{H}), 1.26-1.46(\mathrm{~m}, 6 \mathrm{H}), 0.89(\mathrm{t}, J=7.2 \mathrm{~Hz}, 3 \mathrm{H}) .{ }^{13} \mathbf{C ~ N M R}\left(\mathrm{CDCl}_{3} 100 \mathrm{MHz}\right)$ 202.55, 132.79, 131.11, 130.90, 130.04, 43.67, 32.19, 32.16, 31.46, 28.77, 22.16, 21.49, 13.87. IR (KBr -Neat) 3014, 2957, 2928, 2858, 2717, 1727 (s), 1460, $988 \mathrm{~cm}^{-1}$. EIHRMS calculated for $\mathrm{C}_{13} \mathrm{H}_{22} \mathrm{O} 194.1671$ found 194.1668.

\section{Tetradeca-7,9-dien-1-yne (11)}

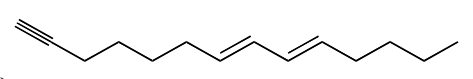

The Ohira-Bestmann reagent ${ }^{10}(3.71 \mathrm{~g}, 19.3 \mathrm{mmol})$ was slowly added to an anhydrous mixture of trideca-6,8-dienal (1.50 g, $7.73 \mathrm{mmol})$ and $\mathrm{K}_{2} \mathrm{CO}_{3}(3.20 \mathrm{~g}, 23.2$ $\mathrm{mmol})$ in methanol $(100 \mathrm{~mL})$ at $0^{\circ} \mathrm{C}$ under nitrogen. The mixture was stirred under nitrogen for $2 \mathrm{~h}$ allowing the reaction to warm to room temperature. At $2 \mathrm{~h}$, all aldehyde was consumed by TLC. The mixture was diluted with ether $(500 \mathrm{~mL})$, washed with 1x200 mL water, $1 \times 100 \mathrm{~mL}$ brine, dried with $\mathrm{MgSO}_{4}$, filtered, and concentrated to an orange heterogeneous oil. The intended alkyne $\mathbf{1 1}$ was isolated as a clear colorless oil $(1.43 \mathrm{~g}, 97 \%)$ in $>98 \%$ purity after silica gel chromatography (100\% pentane) ${ }^{1} \mathbf{H}$ NMR $\left(\mathrm{CDCl}_{3} 400 \mathrm{MHz}\right)_{-}$5.94-6.05 (m, 2H), 5.49-5.62 (m, 2H), 2.19 (dt, J=7.2, $\left.1.2 \mathrm{~Hz}, 2 \mathrm{H}\right)$,

${ }^{10}$ a) Muller, S.; Liepold, B.; Roth, B.; Bestmann, H.J. Synlett. 1996, 521-522. b) Xu, Z.; Peng, Y.; Ye, T. Org. Lett. 2003, 5(16), 2821-2824. 
2.07 (quintet, $J=8.0 \mathrm{~Hz}, 4 \mathrm{H}), 1.94(\mathrm{t}, J=2.8 \mathrm{~Hz}, 1 \mathrm{H}), 1.46-1.58(\mathrm{~m}, 4 \mathrm{H}), 1.26-1.40(\mathrm{~m}$, $4 \mathrm{H}), 0.89$ (t, $J=7.2 \mathrm{~Hz}, 3 \mathrm{H}) .{ }^{13} \mathbf{C}$ NMR $\left(\mathrm{CDCl}_{3} 125 \mathrm{MHz}\right) \ldots 132.70,131.55,130.74$, $130.16,84.52,68.19,32.25,31.99,31.54,28.41,27.92,22.23,18.25,13.93$. IR $(\mathrm{KBr}$ -Neat) $3311,3014,2957,2928,2859,1460,1434,987,629 \mathrm{~cm}^{-1}$. EI-HRMS calculated for $\mathrm{C}_{14} \mathrm{H}_{22} 190.1721$ found 190.1711 .

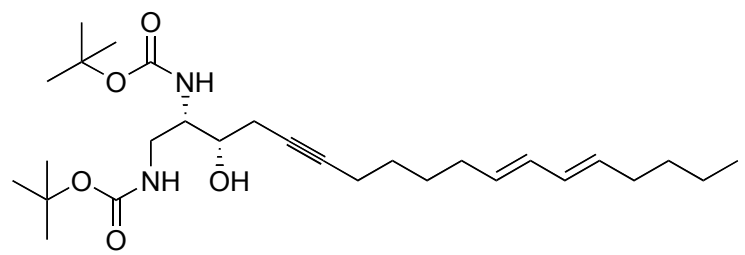

$(2 S, 3 S)-(+)-B i s-1,2-($ tert-butoxycarbonylamino)-3-hydroxyoctadeca-11,13-dien-5-yne (12)

n-Butyllithium (2.48M in hexanes, $1.04 \mathrm{~mL}, 2.58 \mathrm{mmol}$ ) was added dropwise to an anhydrous solution of alkyne $11(492 \mathrm{mg}, 2.6 \mathrm{mmol})$ in toluene $(35 \mathrm{~mL})$ under nitrogen at $-25^{\circ} \mathrm{C}$. The solution was stirred at $-20^{\circ} \mathrm{C}$ for $20 \mathrm{~min}$. The solution was allowed to warm to $0^{\circ} \mathrm{C}$ and stirred at $0^{\circ} \mathrm{C}$ for an additional $5 \mathrm{~min}$. At which point, $\mathrm{Me}_{2} \mathrm{AlCl}(1 \mathrm{M}$ in hexanes, $2.6 \mathrm{~mL}, 2.6 \mathrm{mmol}$ ) was added to the above thick solution at $0^{\circ} \mathrm{C}$. The resulting white cloudy solution was stirred at $0^{\circ} \mathrm{C}$ for $1 \mathrm{~h}$. This solution at $0^{\circ} \mathrm{C}$ was cannulated to an anhydrous solution of epoxide $7(252 \mathrm{mg}, 0.834 \mathrm{mmol})$ in toluene (35 $\mathrm{mL}$ ) cooled to $0^{\circ} \mathrm{C}$ under nitrogen. The cloudy white solution was then stirred at $0^{\circ} \mathrm{C}$ for $2.75 \mathrm{~h}$. A saturated aqueous solution of $\mathrm{NaF}(4.0 \mathrm{~mL})$ was added to the above reaction at $0^{\circ} \mathrm{C}$, and the resulting mixture was stirred for _ $\mathrm{h}$ allowing the mixture to warm to room temperature. The mixture was diluted with methylene chloride $(100 \mathrm{~mL})$, dried with $\mathrm{Na}_{2} \mathrm{SO}_{4}$, filtered, and concentrated to approximately $1 / 2$ of the volume of the original toluene solution. This solution was directly subjected to silica gel chromatography $(20 \%$ ethyl acetate in petroleum ether until the toluene solvent eluted than $25 \%$ ethyl acetate in petroleum ether) to isolate the intended alcohol 12 as a yellow oil $(375 \mathrm{mg}, 91 \%)$. [_] $]_{\mathbf{D}}^{23.1}$ $=+1.1\left(\mathrm{c}=0.90\right.$ in $\left.\mathrm{CHCl}_{3}\right) .{ }^{1} \mathbf{H} \mathbf{~ N M R}\left(\mathrm{CDCl}_{3} 500 \mathrm{MHz}\right)$ _5.96-6.03 (m, 2H), 5.51-5.60 $(\mathrm{m}, 2 \mathrm{H}), 4.91(\mathrm{~s}, 1 \mathrm{H}), 4.85(\mathrm{~s}, 1 \mathrm{H}), 3.76-3.82(\mathrm{~m}, 1 \mathrm{H}), 3.65-3.70(\mathrm{~m}, 1 \mathrm{H}), 3.34(\mathrm{dt}$, $J=14.8,7.5 \mathrm{~Hz}, 1 \mathrm{H}), 3.30(\mathrm{~s}, 1 \mathrm{H}), 3.17-3.23(\mathrm{~m}, 1 \mathrm{H}), 2.42$ (ddt, $J=16.4,7.4,2.3 \mathrm{~Hz}, 1 \mathrm{H})$, 2.35 (ddt, $J=16.6,6.2,2.3 \mathrm{~Hz}, 1 \mathrm{H}), 2.13-2.17$ (m, 2H), 2.02-2.09 (m, 4H), 1.43-1.50 (m, $22 \mathrm{H}), 1.24-1.37(\mathrm{~m}, 4 \mathrm{H}), 0.89(\mathrm{t}, J=7.1 \mathrm{~Hz}, 3 \mathrm{H}) .{ }^{13} \mathbf{C}$ NMR $\left(\mathrm{CDCl}_{3} 125 \mathrm{MHz}\right)_{-} 157.15$, $156.19,132.42,131.56,130.75,130.31,82.56,79.79,79.47,76.09,69.39,53.48,42.29$, $32.12,31.94,31.54,28.58,28.41,28.34,24.23,22.13,18.60,13.75$. IR ( $\mathrm{KBr}-\mathrm{Neat})$ 3361, 2978, 2931, 1692 (s), 1514, 1454, 1392, 1367, 1311, 1251, 1169, 1055, 988, 758 $\mathrm{cm}^{-1}$. EI-HRMS calculated for $\mathrm{C}_{28} \mathrm{H}_{48} \mathrm{~N}_{2} \mathrm{O}_{5} 492.3563$ found 492.3540. EA calculated for $\mathrm{C}_{28} \mathrm{H}_{48} \mathrm{~N}_{2} \mathrm{O}_{5}$ : C: 68.26; $\mathrm{H}: 9.82$ found $\mathrm{C}: 67.90 ; \mathrm{H}: 9.93$. 


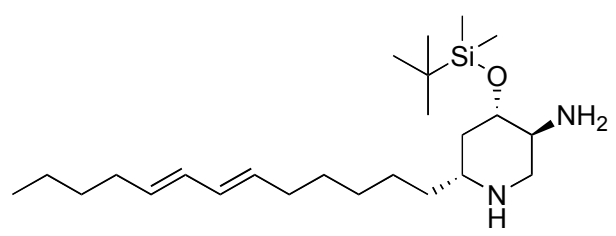

$O$-(tert-Butyl-dimethylsilany)-Pseudodistomin D (13)

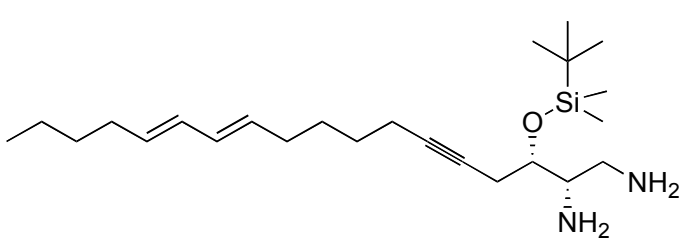

2: 3-(tert-Butyl-dimethylsilanyloxy)octadeca-11,13-dien-5-yne-1,2-diamine: An anhydrous solution of tertbutyldimethylsilyl trifluoromethanesulfonate (77_L, $89 \mathrm{mg}, 34$ _mol) and 2,6-lutidine (42_L, $39 \mathrm{mg}, 36$ mol) in anhydrous methylene chloride $(2 \mathrm{~mL})$ was cannulated to an anhydrous solution of protected diamine $\mathbf{1 2}(22 \mathrm{mg}, 45$ $\mathrm{mol})$ in methylene chloride $(2 \mathrm{~mL})$ under nitrogen. The homogenous and colorless solution was stirred in a sealed tube for $2 \mathrm{~h}$ at $40^{\circ} \mathrm{C}$. The reaction was cooled to room temperature, and the solvent was removed in vacuo. The oily mixture was taken up in a solution of methanol $(3 \mathrm{~mL})$, THF $(1 \mathrm{~mL}), \mathrm{H}_{2} \mathrm{O}(0.5 \mathrm{~mL})$ and acetic acid $\left(60 \_\mathrm{L}\right)$. The solution was stirred in a sealed tube for $2 \mathrm{~h}$ at $50^{\circ} \mathrm{C}$. The reaction was concentrated in vacuo to an oily solid. The material was suspended in $1 \mathrm{M} \mathrm{NaOH}(15 \mathrm{~mL})$ and brine $(15$ $\mathrm{mL})$. This suspension was extracted with $3 \times 15 \mathrm{~mL}$ methylene chloride. The organic portion was dried with $\mathrm{Na}_{2} \mathrm{SO}_{4}$, filtered, concentrated in vacuo, and further dried under high vacuum ( 0.5 torr) overnight to afford the protected diamine $\mathbf{2}$ as a slightly green oil. The material was isolated in approximately $95 \%$ purity by ${ }^{1} \mathrm{H}$ NMR analysis and used in the next step without further purification. ${ }^{1} \mathbf{H}$ NMR $\left(\mathrm{CDCl}_{3} 500 \mathrm{MHz}\right){ }_{-} 5.95-6.04(\mathrm{~m}$, $2 \mathrm{H}), 5.50-5.62(\mathrm{~m}, 2 \mathrm{H}), 3.69-3.73(\mathrm{~m}, 1 \mathrm{H}), 2.78-2.83(\mathrm{~m}, 1 \mathrm{H}), 2.68(\mathrm{dd}, J=12.4,4.3 \mathrm{~Hz}$, $1 \mathrm{H}), 2.58(\mathrm{dd}, J=12.4,8.8 \mathrm{~Hz}, 1 \mathrm{H}), 2.45$ (ddt, $J=16.3,8.7,2.2 \mathrm{~Hz}, 1 \mathrm{H}), 2.29$ (ddt, $J=16.5$, 4.6, 2.1 Hz, 1H), 2.11-2.15 (m, 2H), 2.03-2.08 (m, 4H), 1.20-1.50 (m, 12H), 0.87-0.91 $(\mathrm{m}, 12 \mathrm{H}), 0.09$ (s, 3H), $0.06(\mathrm{~s}, 3 \mathrm{H}) .{ }^{13} \mathbf{C ~ N M R}\left(\mathrm{CDCl}_{3} 125 \mathrm{MHz}\right)+132.62,131.67$, $130.61,130.16,82.32,76.32,72.56,56.44,46.35,32.22,31.99,31.51,28.55,28.35$, $25.76,24.67,22.20,18.60,17.96,13.90,-4.28,-4.89$.

An anhydrous suspension of diamine 2 (all material from above) and AgOTs (1.3 $\mathrm{mg}, 4.7$ mol $)$ in acetonitrile $(2 \mathrm{~mL})$ was stirred at $40^{\circ} \mathrm{C}$ under nitrogen in a sealed tube for $2 \mathrm{~h}$. The resulting yellow homogenous solution was cooled to room temperature and diluted with a solution of methanol $(2 \mathrm{~mL})$, water $(0.5 \mathrm{~mL})$, THF $(1 \mathrm{~mL})$ and acetic acid (7_L, $7.3 \mathrm{mg}, 0.12 \mathrm{mmol}$ ). After stirring for $10 \mathrm{~min}$, the above solution was treated with a solution of $\mathrm{NaBH}_{3} \mathrm{CN}(80 \mathrm{mg}, 1.3 \mathrm{mmol})$ in methanol $(1 \mathrm{~mL})$ to afford a deep tan heterogeneous mixture that was stirred at room temperature for $18 \mathrm{~h}$. The mixture was concentrated in vacuo to a black oily residue. The residue was suspended in $1 \mathrm{M} \mathrm{NaOH}$ $(15 \mathrm{~mL})$ and methylene chloride $(5 \mathrm{~mL})$. The mixture was vigorously stirred for ${ }_{-} \mathrm{h}$, saturated with solid $\mathrm{NaCl}$, diluted with brine $(10 \mathrm{~mL})$ and extracted with $3 \times 20 \mathrm{~mL}$ methylene chloride. The organic extract was dried with $\mathrm{Na}_{2} \mathrm{SO}_{4}$, filtered, and concentrated in vacuo to a black oily residue. Purification by silica gel chromatography (4\% $\mathrm{NH}_{4} \mathrm{OH}$ aq. (30\% $\mathrm{NH}_{3}$ in water), $4 \%$ methanol in ethyl acetate) and high vacuum drying ( 0.5 torr) for $18 \mathrm{~h}$ afforded protected piperidine $\mathbf{1 3}$ as a slightly yellow viscous oil (9.6 mg, 52\% for two steps from 12). [ $]_{\mathbf{D}}{ }^{25}=+15$ (c=0.52 in $\left.\mathrm{CHCl}_{3}\right) .{ }^{1} \mathbf{H ~ N M R}\left(\mathrm{CD}_{3} \mathrm{OD}\right.$ $500 \mathrm{MHz}{ }_{-} 5.93-6.01(\mathrm{~m}, 2 \mathrm{H}), 5.48-5.57(\mathrm{~m}, 2 \mathrm{H}), 3.35$ (ddd, $\left.J=10.9,9.0,4.7 \mathrm{~Hz}, 1 \mathrm{H}\right)$, 
$3.04(\mathrm{dd}, J=12.2,4.5 \mathrm{~Hz}, 1 \mathrm{H}), 2.50-2.57(\mathrm{~m}, 2 \mathrm{H}), 2.35(\mathrm{dd}, J=12.1,11.1 \mathrm{~Hz}, 1 \mathrm{H}), 2.01-$ $2.08(\mathrm{~m}, 4 \mathrm{H}), 1.90(\mathrm{ddd}, J=12.6,4.6,2.5 \mathrm{~Hz}, 1 \mathrm{H}), 1.25-1.50(\mathrm{~m}, 15 \mathrm{H}), 1.11$ (quartet, $J=11.3 \mathrm{~Hz}, 1 \mathrm{H}), 0.89-0.93(\mathrm{~m}, 12 \mathrm{H}), 0.12(\mathrm{~s}, 3 \mathrm{H}), 0.11(\mathrm{~s}, 3 \mathrm{H}) .{ }^{13} \mathbf{C}$ NMR $\left(\mathrm{CD}_{3} \mathrm{OD} 125\right.$ $\mathrm{MHz})_{-} 133.20,133.01,132.13,131.99,77.16,56.80,56.62,51.35,42.03,37.18,33.67$, $33.47,33.01,30.60,30.42,27.05,26.46,23.43,18.98,14.43,-3.69,-4.33$. IR (KBr -Neat) $2927,2856,1462,1252,1089,986,836,775 \mathrm{~cm}^{-1}$. EI-HRMS calculated for $\mathrm{C}_{24} \mathrm{H}_{48} \mathrm{~N}_{2} \mathrm{OSi} 408.3536$ found 408.3542 .

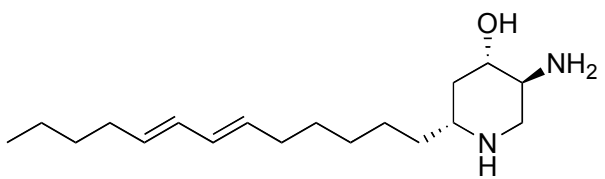

\section{Pseudodistomin D (1)}

A solution of TBS protected alcohol $13(9.2 \mathrm{mg}, 23$ mol) and TBAF (1M in THF, $90 \_$L, $\left.90 \_\mathrm{mol}\right)$ in THF ( $\left.5 \mathrm{~mL}\right)$ was stirred for $20 \mathrm{~h}$ at room temperature. The reaction was directly chromatographed through silica gel $\left(10 \% \mathrm{NH}_{4} \mathrm{OH}\left(30 \% \mathrm{NH}_{3}\right.\right.$ in $\left.\mathrm{H}_{2} \mathrm{O}\right), 10 \%$ methanol in ethyl acetate) and concentrated overnight under high vacuum $(0.5$ torr) to afford pseudodistomin D 1 as a white gum $(7 \mathrm{mg}, 100 \%)$. [ $]_{\mathrm{D}}{ }^{25}=+6(\mathrm{c}=0.2 \mathrm{in}$ $\mathrm{MeOH})$ literature $^{11}\left([]_{\mathrm{D}}{ }^{25}=+5(\mathrm{c}=0.26\right.$ in $\mathrm{MeOH}) .{ }^{1} \mathbf{H}$ NMR $\left(\mathrm{CD}_{3} \mathrm{OD} 500 \mathrm{MHz}\right)$ 5.93-6.01 (m, 2H), 5.47-5.56 (m, 2H), 3.24 (ddd, $J=11.2,9.3,4.6 \mathrm{~Hz}, 1 \mathrm{H}), 3.07$ (dd, $J=12.2,4.5 \mathrm{~Hz}, 1 \mathrm{H}), 2.52-2.58(\mathrm{~m}, 2 \mathrm{H}), 2.35(\mathrm{dd}, J=12.1,11.1 \mathrm{~Hz}, 1 \mathrm{H}), 2.02-2.08(\mathrm{~m}$, 4H), 1.98 (ddd, $J=12.7,4.7,2.5 \mathrm{~Hz}, 1 \mathrm{H}), 1.27-1.47$ (m, 12H), 1.09 (ddd, $J=11.4,11.4$, $11.4 \mathrm{~Hz}, 1 \mathrm{H}), 0.90(\mathrm{t}, J=7.1 \mathrm{~Hz}, 3 \mathrm{H}) .{ }^{13} \mathbf{C} \mathbf{N M R}\left(\mathrm{CD}_{3} \mathrm{OD} 125 \mathrm{MHz}\right){ }_{-} 133.21,132.99$, 132.12, 131.97, 74.99, 56.80, 56.32, 51.39, 41.20, 37.15, 33.66, 33.47, 33.00, 30.61, 30.44, 27.02, 23.42, 14.42. IR (KBr - neat) 3282, 3013, 2925, 2853, 1560-1598, 1465, $984 \mathrm{~cm}^{-1} .{ }^{1} \mathrm{H},{ }^{13} \mathrm{C}$ NMR, and IR data correlates with the listed chemical shifts and coupling constants reported for the isolated material. ${ }^{11}$

Table SI 1: ${ }^{1} \mathrm{H}$ NMR correlation between the synthetic and natural Pseudodistomin D

\begin{tabular}{|c|c|c|c|c|c|c|c|}
\hline carbon & $\begin{array}{c}\text { Isolated } \\
\text { Lit. }^{a}\end{array}$ & Mult. & coupling & Synthetic $^{\mathrm{b}}$ & Mult. & coupling & $\begin{array}{c}\text { Difference } \\
\text { in shift }^{c}\end{array}$ \\
\hline 2 & 2.5 & $\mathrm{~m}$ & & 2.52 & $\mathrm{~m}$ & & -0.02 \\
\hline \multirow[t]{2}{*}{3} & 1.96 & ddd & $4.6,4.6,12.1$ & 1.98 & & $2.5,4.7,12.7$ & -0.02 \\
\hline & 1.07 & ddd & $11.1,11.3,12.1$ & 1.09 & & $11.4,11.4,11.4$ & -0.02 \\
\hline 4 & 3.2 & ddd & $4.6,9.5,11.1$ & 3.24 & & $4.6,9.3,11.2$ & -0.04 \\
\hline 5 & 2.5 & $\mathrm{~m}$ & & 2.52 & $\mathrm{~m}$ & & -0.02 \\
\hline \multirow[t]{2}{*}{6} & 3.04 & ddd & $4.5,12.1$ & 3.07 & & $4.5,12.2$ & -0.03 \\
\hline & 2.31 & ddd & $10.9,12.1$ & 2.35 & & $11.1,12.1$ & -0.04 \\
\hline $1^{\prime}$ & 1.35 & $\mathrm{~m}$ & & 1.35 & & & 0 \\
\hline $2^{\prime}$ & 1.35 & $\mathrm{~m}$ & & 1.35 & & & 0 \\
\hline $3^{\prime}$ & 1.35 & $\mathrm{~m}$ & & 1.35 & & & 0 \\
\hline $4^{\prime}$ & 1.35 & $\mathrm{~m}$ & & 1.35 & & & 0 \\
\hline $5^{\prime}$ & 2.04 & $\mathrm{dt}$ & $7.0,7.1$ & 2.02 & $\mathrm{~m}$ & & 0.02 \\
\hline $6^{\prime}$ & 5.53 & $\mathrm{dm}$ & 14.4 & 5.49 & $\mathrm{~m}$ & & 0.04 \\
\hline $7^{\prime}$ & 5.96 & $\mathrm{dm}$ & 14.4 & 5.94 & $\mathrm{~m}$ & & 0.02 \\
\hline $8^{\prime}$ & 5.96 & $\mathrm{dm}$ & 14.4 & 5.94 & $\mathrm{~m}$ & & 0.02 \\
\hline
\end{tabular}

\footnotetext{
${ }^{11}$ Freyer, A.J.; Patil, A.D.; Killmer, L.; Troupe, N.; Mentzer, M.; Carte, B.; Faucette, L.; Johnson, R.K. J.
} Nat. Prod. 1997, 60, 986-990. 


\begin{tabular}{|c|c|c|c|c|c|c|c|}
\hline $9^{\prime}$ & 5.53 & $\mathrm{dm}$ & 14.4 & 5.49 & $\mathrm{~m}$ & & 0.04 \\
\hline $10^{\prime}$ & 2.04 & $\mathrm{dt}$ & $7.0,7.1$ & 2.02 & $\mathrm{~m}$ & & 0.02 \\
\hline $11^{\prime}$ & 1.35 & $\mathrm{~m}$ & & 1.35 & & & 0 \\
\hline $12^{\prime}$ & 1.35 & $\mathrm{~m}$ & & 1.35 & & & 0 \\
\hline $13^{\prime}$ & 0.9 & $\mathrm{t}$ & 7.1 & 0.9 & $t$ & 7.1 & 0 \\
\hline
\end{tabular}

Table SI 2: ${ }^{13} \mathrm{C}$ NMR correlation between the synthetic and natural Pseudodistomin D

\begin{tabular}{cccc} 
carbon & Isolated $^{a}$ & Synthetic $^{b}$ & Difference $^{c}$ \\
\hline 2 & 56.7 & 56.8 & -0.1 \\
3 & 41.2 & 41.2 & 0 \\
4 & 75.5 & 75 & 0.5 \\
5 & 56.4 & 56.3 & 0.1 \\
6 & 51.9 & 51.4 & 0.5 \\
$1^{\prime}$ & 37.2 & 37.2 & 0 \\
$10^{\prime}$ & 33.5 & 33.6 & -0.1 \\
$11^{\prime}$ & 32.8 & 33 & -0.2 \\
$12^{\prime}$ & 23.3 & 23.4 & -0.1 \\
$13^{\prime}$ & 14.3 & 14.4 & -0.1 \\
$2^{\prime}$ & 26.9 & 27 & -0.1 \\
$3^{\prime}$ & 30.3 & 30.4 & -0.1 \\
$4^{\prime}$ & 30.5 & 30.6 & -0.1 \\
$5^{\prime}$ & 33.3 & 33.5 & -0.2 \\
$6^{\prime}$ & 132.9 & 133 & -0.1 \\
$7^{\prime}$ & 132 & 132.1 & -0.1 \\
$8^{\prime}$ & 131.8 & 132 & -0.2 \\
$9^{\prime}$ & 133.1 & 133.2 & -0.1 \\
\hline${ }^{\prime}$ & Proton NMR chemical analysis of the isolated Pseudodistomin D \\
as reported by Freyer et. al in CD OD at 100 Mhz. $^{1{ }^{\prime}}$ b $125 \mathrm{Mhz}$ in \\
CD $_{3}$ OD. ${ }^{\prime}$ Difference in chemical shift between the isolated and \\
synthetic pseudodistomin D.
\end{tabular}

